Nutr. Dieta 1965;7:I-IV

\title{
Contents, Vol. 7, 1965
}

\section{Nutritio et Dieta}

Europäische Zeitschriit für Ernährung und Diätetik

European Review of Nutrition and Dietetics

Revue européenne de nutrition et de diététique

EDITORES

E, J. BIGWOOD, Bruxelles H. H. BERG, Hamburg R. BOLLER, Wien

E. LE BRETON, Paris

L. BRULL, Liège

F. S. P. VAN BUCHEM, Haarlem

M. COPPO, Modena

H. D. CREMER, Giessen CH. DARNAUD, Toulouse J. DELFOSSE, Bruxelles M. DEMOLE, Geneve M. J. L. DOLS, Wassenaar

G. DUCHESNE, Paris

H. GLATZEL, Dortmund

W. GLOOR-MEYER, Zurich

J. GONTZEA, Bucarest

H. GOUNELLE, Paris

W. HALDEN, Graz

C. DEN HARTOG, Den Haag

R. JACQUOT, Paris

H. KRAUT, Dortmund

J. KÜHNAU Hamburg J. LEDERER, Louvain

F. MANCINI, Roma

A. P. MEIKLEJOHN, Edinburgh

H. G. MOGENA, Madrid

A. MOSSE, Paris

R. NICOLAYSEN, Oslo

G. PEQUIGNOT, Paris

H. SARLES, Marseille

T. F. S. M. VAN SCHAIK, Den Haag

G. S. SCHETTLER, Berlin

A. VAN DER SCHUEREN, Gand

H. M. SINCLAIR, Oxford

J. C. SOMOGYI, Zurich

F. J. TRAISSAC, Bordeaux

L. TRAVIA, Roma

G. P. VECCHI, Modena

G. VERDONK, Gand

E. VERZAR, Basel 
R. WENGER, Wien

REDACTORES

E. AZERAD, Paris

J. TREMOLIERES, Paris

H. KAPP, Basel

K. A. J. WRETLIND, Stockholm

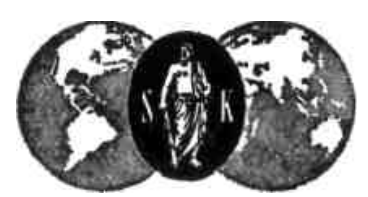

1965

Vol. 7

BASEL (Schweiz)

S. KARGER

NEW YORK

INDEX

Apfelbaum, M. et Trémolières, J.: Dépenses énergétiques et masse active chez Tobèse. Energy Output and Active Mass in the Obese. - Kalorienverbrauch und aktive Masse beim Fettsüchtigen 241

Atanackovic, V.; Gjivanovic, B. and Jovanovic, V.: Absorption and Excretion of Fat in Cases of Pellagra. - Fettresorption und -exkretion beí Pellagra. - Absorption et excretion des graisses dans la Pellagre 186

Barbaresi, F.: vide Rinetti, M.

Blumberger, W. und Glatzel, H.: Beiträge zur Physiologie der Gewürze und Würzstoffc. V. Die Speichelwirksamkeit der Paprika. - The Physiological Effects of Spices and Con diments. V. Salivary Effects of Paprika. - Contributions à la physiologie des épices et aromates. V. Activité salivaire du paprika 222

Bobek, P.: vide Ginter, E.

Braham, J. E.: vide Bressani, R.

Bressani, R.; Braham, J. E.; Elias, L. G. and Zaghi, Silvia de: Relationship Between

Net Protein Utilization (NPU) and Nitrogen Efficiency Ratio (NER). - Bezíehung zwíschen Eiweissnutzwert und Wachstumsuoert. - Relation entre Futilisation nette des protéines (NPU) et le rapport d'efficience azotée (NER) 161

Canzler, H. und Glatzel, H.: Beiträge zur Physiologie der Gewürze und Würzstoffe.

VI. Aktivierung der Nebennierenrindenfunktion durch Capsicum-Gewürze? - The Physiological Effects of Spices and Condiments. VI. Do Capsicum Peppers Stimulate Adrenocortical Function? - Contribution à la physiologie des épices et aromates. VI. Activation des fonctions surrénales par les racines de capsicum ? 303

Cassan, A. et Derache, R.: Bilan azote et excretion urinaire des glycoprotéines chez le rat en regeneration hépatique. - Srickstoffhílanz und Glukoproteinausscheidung im Rattenurin beí Leberzellregeneration. - Nitrogen Balance and Urinary Glycoprotein Excretion in the Rat During Liver Regeneration 108

Colombi, L.: vide Rinetti, M.

Cresta, M. et Passarello, P.: Composition du corps, proportions somatiques et métabolisme de base dans Гâge post-pubéral. - Body Composition, Somatic Proportions and 
Basal Metabolic Rate in the Post-Puberal Age. - Zusammensetzung des Körpers, Kòrperproportionen und Grundumsatz im post-puberalen Alter 255

Dalderup, L.M.; Hartog, C. den ; Pol, G. and Rutgers, R.: Studies in Rats Given a

Barley Product Mixed up in Food. Growth, Fertility, Stress, Intestinal Function,

Longevity. - Untersuchungen über die Wirkung eines Gerstenproduktes als Zugabe zur

Nahrung beí Ratten auf Wachstum, Fertílítät, Stress, Darmfunktíon und Lebensdauer. -

Etude sur des rats recevant un produit à base d'orge mêlé à leurs aliments. Croissance,

fertilité, «stress», fonctions intestinales, longévité 120

Derache, R.: vide Cassan, A.

Droste, H. und Jekat, F.: Nährstoffversorgung in Arbeitnehmer- und Rentnerhaushalten der Bundesrepublik Deutschland während der Zeit von 1950-1962. II. Die Zufuhr von Mineralien und Ballaststoffen, Vitaminen und Aminosäuren. - Supply in Nutrients in Workers' 1 and Pensioners'1 Families in the Federal German Republic 1950-1962. II. Supply of Minerals and Ballast, Vitamins and Amino Acids. - Alimentation des families de travailleurs et de pensionnés en République Fédérale Allemande de 1950-1962. II.

Apports en minéraux, substances de ballast, vitamines et acides amines

1

Dworschâk, E.: vide Tarján, R.

Elias, L. G.: vide Bressani, R.

Gerbelová, M.: vide Ginter, E.

Ginter, E.; Bobek, P. and Gerbelová, M.: The Influence of Scorbut and Prolonged Low

Intake of Vitamin C on Serum Lipoproteins in Guinea-Pigs. - Der Eínfiuß von Skorbut

und langfristiger Vitamin-C-Unterernährung auf die Serum-Lípoproteíne von Meer-

schweinchen. - L'influence du scorbut et de Гapport bas prolongé de vitamine $\mathrm{C}$ sur les

lipoprotéines sériques chez les cobayes

103

Gjivanovió, B.: vide Atanackovic, V.

Glatzel, H. und Rettenmaier, G.: Ernährungsphysiologische Brotvergleiche. VII. Der Verlauf der

alimentären Hyperglykämie nach Brotkruste (Rinde) und Brotkrume. -

Comparison of Various Breads in the Physiology of Nutrition. VII. The Course of

Nutritional Hyperglycaemía after Crust and Crumb. - Comparaisons de physiologic

alimentaire avec le pain. VII. Evolution de Thyperglycémie alimentaire après con-

sommation de la croûte ou de la mie de pain 51

Glatzel, H.: vide Blumbekgeb, W.

Glatzel, H.: vide Canzler, H.

Guggenheim, K.: vide Tal, E.

Hahn, P.: vide Melichar, V.

Håkansson, I.: Effects in Dogs of Two Fat Emulsions for Intravenous Nutrition. - Wír-

kungen zweíer Fettemulsionen für intravenöse Ernährung beím Hund. - Effets de deux

emulsions de graisse pour Talimentation intra-veineuse chez les chiens

90

Hartog, C. den: vide Dalderup, L. M.

Hejda, S.: vide Osancová, K.

Isaksson, B.; Sjögren, B. with the technical assistance of Weimers, G., Dietician: On the Concept "Constant Diet" in Metabolic Balance Studies. - Uber den Begriff «konstante Kost» beí Stojfwechselbilanzuntersuchungen. - Sur le concept «diète constante» dans les etudes de balance métabolique $\quad 175$

Jekat, F.: vide Droste, $\mathrm{H}$.

Jekat, F.: vide Zimmermann-Telschow, $H$. 
Jovanovic, V.: vide Atanackovic, V.

Koldovsky, O.: vide Melichar, V.

Kkámer, M.; Szöke, K.: Lindner, K. and Tarján, R.: The Effect of Different Factors on the Composition of Human Milk and its Variations. III. Effect of Dietary Fats on the Lipid Composition of Human Milk. - Wírkung verschiedener Faktoren auf die Zusammensetzung menschlícher Milch. III. Wírkung des Nahrungsfettes auf den Fettgehalt der menschlichen MíL·h. - Effet de différents facteurs sur la composition du lait et humain ses variations. III. Effet des graisses alimentaires sur la composition enlipides du lait humain $\quad 71$

Kramer, Magdalene : vide Tarján, R.

Latham, M. C. and Robson, J. R. K.; A Trial to Evaluate the Benefit of Different Protein Rich Foods to African School Children. - Bewertungsversuch des Nutzens verschiedener eíweíßreícher Nahrungsmíttel bei afríkaníschen Schulkíndern. - Essai d'apprêciation de la valeur de différents aliments riches en protéines chez les écoliers africains 28

Lindner, K.: vide Kramer, M.

Lindner, K.: vide Tabján, R.

Melichar, V.; Novak, P.; Novak, M.; Hahn, P. and Koldovsky, O.: Blood Cholesterol Levels in Newborn Infants and Adults in Ulan Bator (Mongolia) and Prague (Czechoslovakia). - BlutCholesterínwerte beí Neugeborenen und Erwachsenen in Ulan Bator (Mongolei) und in Prag (Tschechoslowakei). - Taux du cholesterol sanguin chez les nouveaux-nés et les adultes d'Ulan Bator (Mongolie) et de Prague (Tchécoslovaquie) 191

Novak, M.: vide Melichar, V.

JN ovák, P.: vide Melichar, V.

Osancová, K. and Hejda, S.: Incidence of Overweight in Czechoslovakia. - Häufigkeít von Übergewichtígkeít in der Tschechoslowakei. - Frequence de Гobésité en Tchécoslovaquie 211

Parizková, J.; Stanková, L; §prynarová, S. et Vambebová, M.: Influence de Гexercice physique sur certains index métaboliques sanguins chez les garçons obèses après $\Gamma$ effort. Influence of Physical Exercice on Certain Hematometabolíc Features in Obese Boys. Wirkung körperlicher Arbeit auf gewisse Stoffwechselindexe bei Fettsüchtigen im Kindesalter 21

Passabello, P.: vide Cbesta, M.

Placeb, Z.: vide Slabochová, Z.

Pol, G.: vide Daldebup, L. M.

Rath, R.: vide Slabochová, Z.

Rettenmaier, G.: vide Glatzel, H.

Rinetti, M.; Visioli, O.; Colombi, L. and Babbabesi, F.: The Cardial Lipids after Intense Muscular Work in Rats Fed on a Diet Lacking Essential Fatty Acids. - Die Fette des Rattenherzens nach íntensiver Muskelarbeít beí einer Kost ohne ungesättígte Fettsäuren. - Les lipides cardiaques après un travail musculaire intense chez les rats soumis à un regime prive d'acides gras essentiels 81

Robson, J. R. K.: vide Latham, M. C.

Rutgers, R.: vide Dalderup, L. M.

Sjögren, B.: vide Isaksson, B.

Slabochová, Z.; Rath, R. and Placer, Z.: Effect of Methandrostenolon and Dimethandrostenolon in the Dietotherapy of Obesity. - Wirkung von Methandrostenolon und 
Dimethandrostenolon in der Behandlung der Fettsucht. - Effets du méthandrostenolone et du diméthandrostanolone dans le traitement diétique de Гobésité 271

Somogyi, J. C.: vide Trautner, K.

Sprynarová, S.: vide Parizkovâ, J.

Stanková, L.: vide Parizkovâ, J.

Szarvas, T.: vide Tarján, R.

Szöke, K.: vide Kramer, M.

Szöke, K.: vide Tarján, R.

Tal, E. and Guggenheim, K.: Effect of Copper and Manganese on Calcification of Bones of Rats Fed on Meat. - Wírkung von Kupfer und Mangan aufdie Verkalkung der Knochen von Ratten bei Fleíschfutterung. - Effet du cuivre et du manganese sur la calcification des os chez les rats nourris avec de la viande $\quad 62$

Tarján, R.; Kramer, Magdalene ; Szöke, K.; Lindner, K.; Szarvas, T. and Dworschák, E.: The Effect of Different Factors on the Composition of Human Milk. II. The Composition of Human Milk During Lactation. - Wirkungen verschíedener Faktoren auf die Zusammensetzung der menschlichen Milch. II. Zusammensetzung der menschlíchen Mïlch während der Stíllperiode. - Effet de différents facteurs sur la composition du lait humain. II. Composition du lait humain pendant la lactation 136

Tarján, R.: vide Kramer, M.

Trautner, K. und Somogyi, J. C.: Biosynthese von Vitamin C in Kartoffelscheiben. Biosynthesis of Vitamin C in Potato Slices. - Biosynthese de la vitamine C dans les tranches de pommes de terre 196

Trémolières, J.: vide Apfelbaum, M.

Vamberová, M.: vide Parizkovâ, J.

Visioli, O.: vide Rinetti, M.

Weimers, G.: vide Isaksson, B.

Zaghi, Silvia de : vide Bressani, R.

Zimmermann-Telschow, H.: Die Veränderung der freien Aminosäuren im Nüchternblut in Abhängigkeit von der Ernährung beim Menschen. II. Ernährungsversuche mit Milchprotein. - Nutritionally Dependent Changes in Free Amino Acids in Human Fasting Blood. II. Nutritional Studies with Milk Protein. Modification des acides amines libres du sang à jeun en fonction de l'alimentation chez Thomme. II. Experiences avec les protêines du lait 37

Zimmermann-Telschow, H. und Jekat, F.: Die Veränderung der freien Aminosäuren im Nüchternserum und im Harn in Abhängigkeit von der Ernährung beim Menschen. III. Ernährungsversuche mit Weizenbrot. - Changes in the Free Amino Acids in Fasting Serum and Urine of Human Subjects in Relation to Diet. III. Experimental Feeding with Wheat Bread. Modification des acides amines libres du serum à jeun et de l'urine en relation avec l'alimentation chez l'homme. III. Etudes sur le pain de froment .... 283

Buchbesprechungen - Book Reviews - Livres Nouveaux $\quad 80,155,238,315$

Erratum 240

Varia 160

Alle Rechte, insbesondere das der Übersetzung in frerade Sprachen, vorbehalten.

Ohne ausdrückliche Genehmîgung des Verlages ist es auch nicht gestattet, diesen Band Oder Teile

daraus auf photomechanischem Wege (Photokopie, Mikrokopie) zu vervielfältigen. 
(C)

Copyright 1965 by S. Karger AG, Basel

Printed in Switzerland

Cliches: Aberegg-Steiner \& Cie., AG

Druck: Lüdin AG, Liestal 